\title{
Mechanisms for turn alternation in woodlice (Porcellio scaber): The role of bilaterally asymmetrical leg movements
}

\author{
R. N. HUGHES \\ University of Canterbury, Christchurch, New Zealand
}

\begin{abstract}
Angles of free turn were observed in woodlice (Porcellio scaber) after they had been forced to turn left or right in runways. Alternation angles were increased by greater forced-turn angles and number of successive forced turns in one direction. Removal of one or both antennae had no effect on alternation. It was also repeatedly observed that the closer a woodlouse remained to the far wall while traveling along the exit alley of a runway, the greater was its angle of alternation. However, orientation toward this far wall bore no relation to alternation. When given three successive left or right forced turns followed by a fourth in the opposite direction, woodlice moved further away from the exit-alley far wall and repeated the last forced turn at the choice area. Along with results of the other experiments, this result in particular supported an explanation for woodlouse alternation based on bilaterally asymmetrical leg movements (BALM) arising from the negotiation of forced turns. Such asymmetry is seen as biasing an animal to turn in the opposite direction to a preceding forced turn.
\end{abstract}

The tendency to alternate or turn in the opposite direction to a preceding turn, shown by a number of arthropod species, enables corrections for deviations from a linear path of movement (Dingle, 1965; Kupferman, 1966; Hughes, 1978). Unlike spontaneous alternation observed in rats and other vertebrates (Dember, 1961), most arthropod alternation is generated by response rather than environmental stimulus cues (although Wilson \& Fowler, 1976, have observed stimulus alternation in the cockroach, Blatta orientalis). Corballis and Beale (1983) have discussed arthropod alternation within a broader context by suggesting that it may reflect an innate ability to perform left-right response differentiation since the animal turns left or right in the absence of any external directional cues. In the case of the sowbug or woodlouse (Porcellio scaber Latr.), Hughes $(1967,1978)$ proposed that sequential turn alternation promotes survival by ensuring efficient movement from a hazardous area to one that is more favorable. To support this view, he described greater woodlouse alternation after exposure to a dehydrating environment (Hughes, 1967) and following $72 \mathrm{~h}$ of food deprivation (Hughes, 1978). Woodlice also display significant sequential alternation when confronted with a series of either $\mathrm{T}$ choice points in a maze (Hughes, 1967) or obstacles in a simulated natural environment (Hughes, 1978).

Following dissatisfaction with centrifugal swing accompanied by thigmotaxis (Jensen, 1959), the Hullian concept of reactive inhibition was proposed as the likely mechanism underlying arthropod alternation (Grosslight

The author's mailing address is: Department of Psychology, University of Canterbury, Christchurch 1, New Zealand.
\& Harrison, 1961). But, because some later research failed to support this view (e.g., Dingle, 1964a, 1964b), alternative explanations involving memory were provided by at least two authors (Dingle, 1964b; Kupferman, 1966). However, Hughes $(1967,1978)$ proposed a more parsimonious explanation by suggesting, without empirical evidence, that alternation in P. scaber might arise from relatively more stimulation of legs on the side of the body, which have to travel further and work harder while negotiating a turn. On reaching a T choice-point, the animal would be temporarily biased to turn in the direction opposite to that of the forced turn because of a greater overall influence of the relatively "rested" legs on the opposite side of the body. This possibility was supported by Beale and Webster (1971), who demonstrated subsequent turning in a T-maze in the direction of additional unilateral forced walking. Their result is generally regarded as having confirmed Hughes's hypothesis (see, e.g., Hansell \& Aitken, 1977).

If, following a forced turn, bilaterally asymmetrical leg movements (BALM) encourage a woodlouse to return to its original linear path of movement, evidence of a bias to turn in the opposite direction should be apparent before it reaches a free-turn choice point. It is reasonable that, while the woodlouse moves along the post-forcedturn alley, any asymmetry might appear as (1) variations in orientation away from a line of movement parallel with the walls, or (2) variations in distance from the far wall. Although apparent wall-following behavior might be an inevitable consequence of a BALM-elicited bias, this does not necessarily mean that woodlouse alternation is due merely to a thigmotaxis-related tendency to turn in the same direction as the followed wall. Nevertheless, since woodlice display thigmotactic behavior (Pardi \& Papi, 
1961), a wall-following explạnation should not be ignored, even though thigmotaxis seems unimportant for alternation in the related pill bug, Armadillidium vulgare (Kupferman, 1966) and in certain insects (Dingle, 1961, 1964a, 1964b; Grosslight \& Harrison, 1961).

In most studies of arthropods, alternation has been determined by left or right turns following a forced turn. In addition to recording whether or not $A$. vulgare alternated, Kupferman (1966) was able to estimate the magnitude of the tendency for each animal by recording the angle of its free turn. Experiments in the present paper were therefore mainly concerned with examining the contribution to free-turn angle of behavior occurring between forced and free turns with a view to determining whether or not woodlouse alternation really does arise from BALM. In particular, it was intended to determine the role of thigmotaxis-related wall-following behavior. This was necessary since (1) any contribution of thigmotaxis to woodlouse alternation has never been decisively eliminated, and (2) the apparently conclusive results of Beale and Webster (1971) supporting BALM could have arisen from subsequent orienting toward the wall of the maze stem on the same side as the additional forced walking. Their subjects were required to negotiate a treadmill moving against the direction of travel of legs on one side of the body. Because of a bilateral difference in resistance to forward movement, this procedure may have skewed the whole animal toward the wall on the stimulated side. If it were then to merely follow this wall it would inevitably turn at the choice-point in the direction of the prior forced walking.

\section{EXPERIMENT 1}

Although free-turn angle is directly dependent on forced-turn angle in the related isopod $A$. vulgare (Kupferman, 1966), a similar relationship has not been formally documented for $P$. scaber. As forced-turn angle was to be utilized in later experiments, it was clearly necessary to establish its influence before attempting to analyze behavior emitted between forced and free turns.

\section{Method}

Subjects. All subjects observed in the present and subsequent experiments were collected from garden refuse and kept in a covered plastic container. They were provided with soil and leaf litter and were fed on sliced carrot. Their environment was kept reasonably humid through the presence of frequently moistened pieces of cotton cloth (which they also ate!). The woodlice were identified as $P$. scaber by means of a taxonomic key developed for New Zealand terrestrial isopods (Hurley, 1950). Sixty woodlice were used in Experiment 1.

Apparatus. The apparatus comprised 10-mm-wide runways cut from 6-mm-thick clear Perspex sheeting with a forced-turn angle of $45^{\circ}, 90^{\circ}$, or $135^{\circ}$. Lengths of the start, or pre-forced-turn, alley and the exit, or post-forced-turn, alley were 60 and $30 \mathrm{~mm}$, respectively. Each runway was positioned within a 200 -mm-diam circle (drawn on white paper) so that the center of the circle was exactly in the middle of the runway's open-ended exit. To prevent escape before the exit was reached, a thin sheet of clear Perspex was placed over each runway. Room fluorescent lighting, approx- imately $2.5 \mathrm{~m}$ above the apparatus, provided even, cool illumination.

Procedure. Each subject was individually introduced into the apparatus at the beginning of the start alley. The Perspex cover was replaced and it was noted exactly where the subject crossed the circumference of the circle $(r=100 \mathrm{~mm})$ after leaving the runway. This point was used to determine the angle of emergence (i.e., free turn-angle) with respect to the middle of the runway exit. Free turns in the same direction as a forced turn were recorded as negative angles. Twenty woodlice experienced each forced turn angle; half in each group were forced to turn left, and half were forced to turn right.

\section{Results and Discussion}

As shown by an ANOVA, free-turn angle increased with greater forced-turn angles $[F(2,57)=12.19$, $\mathrm{p}<.001$; see Figure 1]. The number of animals (out of 20) that turned in the opposite direction (i.e., alternated) following a forced turn of $45^{\circ}, 90^{\circ}$, or $135^{\circ}$ were 14 , 19 , and 20 , respectively. According to two-tailed binomial tests, these numbers were significant following a $90^{\circ}$ $(\mathrm{p}<.002)$ or a $135^{\circ}(\mathrm{p}<.002)$ turn but not a $45^{\circ}$ turn $(\mathrm{p}=.116)$. The difference between the number of alternating subjects forced $45^{\circ}$ and the combined numbers of those forced $90^{\circ}$ or $135^{\circ}$ was significant $\left[\chi^{2}(1)=7.30\right.$, $\mathrm{p}<.01$; the results of the latter two groups were combined to avoid invalidation of the chi-square test by excessively low expected frequencies; Siegel, 1956]. It is clear that, in woodlice, both the tendency to alternate and the magnitude of the response are directly dependent on the angle of a preceding forced turn. As the work involved in negotiating a forced turn would depend on the size of its angle, this result is consistent with a BALM explanation. However, Kupferman (1966) preferred to interpret his result with the related pillbug as evidence that specific information about the forced turn was "remembered" until the free-turn response was made, but did not suggest why the animal should choose to alternate even if it had retained this information.

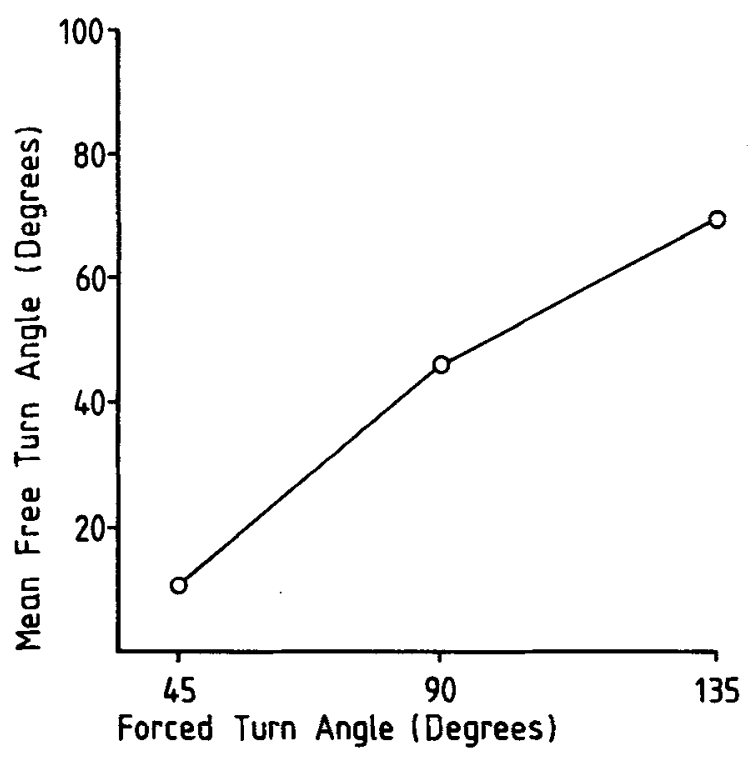

Figure 1. Effects of forced-turn angle on free-turn angle. 


\section{EXPERIMENT 2}

If BALM was responsible for the results of Experiment 1 , the subjects' inner relatively "rested" legs should exert a greater influence on locomotion than the outer legs following a forced turn. This might produce a tendency to walk slightly "crab-wise" at an angle to the far wall of the exit alley. Experiment 2 therefore aimed to establish whether or not there was any relationship between this orientation angle and the angle subsequently turned on reaching the exit.

\section{Method}

Subjects and Apparatus. Twenty-four naive woodlice were observed in this experiment. A small spot of white, water-based fluid used for correcting typing errors ("Snopake') was applied to the central lobe of each subject's trilobed head and, posteriorly, to the median process of its telson. To provide post-forced-turn orientation angles large enough to be measured, all animals were run in the runway with the forced turn of $135^{\circ}$ described in the last experiment. On the section of paper underlying the exit alley were drawn a series of parallel black lines $1 \mathrm{~mm}$ apart. Each trial was recorded on videotape by means of an Hitachi VT-8000 videorecorder and a National WV-361N television camera vertically positioned $100 \mathrm{~mm}$ above the exit alley, which was illuminated by normal room fluorescent lighting.

Procedure. Twelve subjects were individually forced left and 12 were forced right, as described in Experiment 1. After the freeturn angle was noted, a video record of each trial was played back and the image was stopped at distances of 15 and $30 \mathrm{~mm}$ after the forced turn. A transparent overlay was then placed over the 125 mm screen of the camera monitor and a line was drawn along the midline of the subject's image between the anterior and posterior white dots. The angle of intersection of this line with one of the lines on the alley floor comprised the measure of orientation toward the far wall (see Figure 2). Orientations to the near wall were recorded as negative angles.

\section{Results and Discussion}

As there was no significant difference between orientation angles measured $15\left(\right.$ mean $=6.85^{\circ}$ ) and $30 \mathrm{~mm}$ after the forced turn [mean $=5.48^{\circ} ; \mathrm{t}(23)=1.66$, $\mathrm{p}>.1$, average angles for the two distances were correlated with free-turn angles (mean $=85.38^{\circ}$ ). The correlation was not significant $[\mathrm{r}(22)=0.16, \mathrm{p}>.2]$. However, significantly more woodlice were oriented toward the far wall at both post-forced-turn distances $(n=18)$ than were away from it at either distance $(n=6, p=.022)$. There was also no significant difference in free-turn angle between those that were oriented away from (mean $=76.75^{\circ}$ ) and those that were oriented toward the far wall [mean $=88.25^{\circ} ; \mathrm{t}(22)=.73$, $\mathrm{p}>.2]$. All but one subject alternated at the choice area (p < .002).

Although the lack of any relationship between angle of orientation to the exit-alley far wall and free-turn angle was contrary to BALM-derived expectations, it is possible that BALM effects were insufficiently powerful to be detected with the procedure adopted.

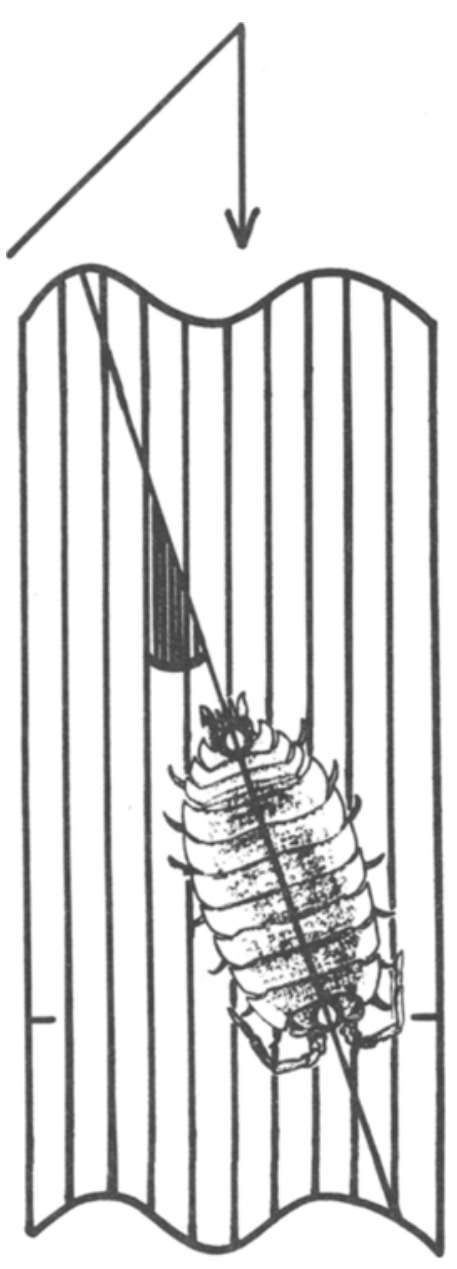

Figure 2. Procedure for measuring the angle of orientation to the far wall of an exit alley.

\section{EXPERIMENT 3}

During the last experiment, many woodlice appeared to remain very close to the far wall while traveling along the exit alley. It therefore seemed likely that, although BALM effects might not noticeably influence orientation to the exit-alley far wall, they might determine how closely woodlice appeared to follow it, since a greater influence of the inner "rested"' legs should force subjects against this wall. In Experiment 3, distance from the far wall was, accordingly, measured and related to free-turn angle following one of two forced-turn angles.

\section{Method}

Subjects and Apparatus. The subjects were 40 naive woodlice. The apparatus comprised the runways with forced-turn angles of $45^{\circ}$ and $135^{\circ}$ described in Experiment 1 and the video-recording equipment described in Experiment 2, except that a 575-mm monitor was used. 
Procedure. Twenty woodlice were individually run through each runway (half forced left, half forced right), and each subject's freeturn angle was noted. By means of the video-recording and playback procedure described in Experiment 2, distances between the outer side of the body and the far wall were measured at points 0,15 , and $30 \mathrm{~mm}$ past the forced turn.

\section{Results and Discussion}

As expected from the results of Experiment 1, the difference between free-turn angles following the two forced-turn angles was significant $\left[45^{\circ}\right.$ mean $=23.98^{\circ}$; $135^{\circ}$ mean $\left.=109^{\circ} ; \mathrm{t}(38)=5.88, \mathrm{p}<.001\right]$. Although the differences between distances from the exit-alley far wall measured at $0 \mathrm{~mm}$ (mean $=1.73 \mathrm{~mm}), 15 \mathrm{~mm}$ $($ mean $=1.58 \mathrm{~mm})$, and $30 \mathrm{~mm}($ mean $=1.88 \mathrm{~mm})$ after the forced turn were not significant, they were suggestive $[F(2,76)=2.53, p>.05<.1]$. This result indicated that subjects moved closest to the wall near the middle of the alley and then moved back again on approaching the exit. Overall, they remained significantly closer to the wall after a $135^{\circ}$ turn (mean $=1.48 \mathrm{~mm}$ ) than after one of $45^{\circ}$ [mean $=1.97 \mathrm{~mm} ; \mathrm{F}(1,38)=5.83$, $\mathrm{p}<.025]$. There was also a significant negative correlation, for all subjects between distance from the wall and free-turn angle $[\mathrm{r}(38)=-.56, \mathrm{p}<.001]$. This means that irrespective of which group they were in, the closer woodlice were to the wall, the greater their free-turn angle.

The number of woodlice (out of 20) that alternated following the $45^{\circ}$ forced turn was not statistically significant $(14, p>.1)$, whereas none of those forced $135^{\circ}$ failed to alternate $(p<.002)$. The subjects that alternated after the forced $45^{\circ}$ turn remained significantly closer to the exit-alley far wall $(n=14$, mean $=1.69 \mathrm{~mm})$ than did those that did not $[\mathrm{n}=6$, mean $=2.61 \mathrm{~mm} ; \mathrm{t}(18)=2.24$, $\mathrm{p}<.05]$.

The main results of this experiment show that the angle of a forced turn determined how close to the far wall woodlice remained while traveling along the exit alley. Distances from the wall were also related to whether or not an individual alternated and to the size of its free-turn angle. These results were consistent with turn alternation arising from effects of BALM at the forced turn.

\section{EXPERIMENT 4}

Earlier research has shown that the number of woodlice that alternate at $\mathrm{T}$ choice-points increases with greater numbers of consecutive forced turns in one direction (Hughes, 1967; Sachs, Klopfer, \& Morrow, 1965). This effect also characterized $T$. molitor larvae (Grosslight \& Ticknor, 1953) and the planarian, Dugesia tigrina (Shinkman \& Hertzler, 1964). In view of the Experiment 3 results, a relationship would be expected between the number of forced turns, distance from the exit-alley far wall, and free turn angle if BALM were the responsible mechanism. Experiment 4 was therefore designed to investigate this possibility with a view to further supporting the role of BALM in woodlouse alternation.

\section{Method}

Subjects and Apparatus. The subjects were 90 naive woodlice run through Perspex runways of the same width and depth as those used in earlier experiments. Each runway had 1, 2, or 3 consecutive $90^{\circ}$ left or right forced turns prior to the exit. In all cases, the length of the alley preceding the first forced turn was $20 \mathrm{~mm}$. The distance between the last forced turn and the choice area was $45 \mathrm{~mm}$. All other alley lengths were $30 \mathrm{~mm}$. The video-recording equipment described in Experiment 3 was also used in this experiment.

Procedure. Equal numbers of subjects (half forced left, half forced right) were individually introduced into each runway. For each animal, the free-turn angle was noted and then, by means of the video-recording and playback procedure described in Experiment 3, distances between the outer side of the body and the exit-alley far wall were measured at points $15,25,35$, and $45 \mathrm{~mm}$ past the last forced turn.

\section{Results and Discussion}

Free-turn angle significantly increased with the number of forced turns $[F(2,87)=6.31, p<.01$; see Figure 3]. Significant numbers of woodlice (out of 30 ) alternated after one $(24, \mathrm{p}<.002)$, two $(28, \mathrm{p}<.001)$, and three forced turns $(28, \mathrm{p}<.001)$. However, because of a ceiling effect, there was no significant difference between the number forced once and the combined numbers forced twice or three times $\left[\chi^{2}(1)=2.38, p>.1\right]$.

Although the number of forced turns did not significantly affect overall distances from the exit-alley far wall $[1$ turn mean $=1.81 \mathrm{~mm} ; 2$ turns mean $=1.63 \mathrm{~mm} ; 3$ turns mean $=1.77 \mathrm{~mm} ; \mathrm{F}(2,87)=0.72, \mathrm{p}>.25$ ], there were slight, but significant, differences between distances measured at the four points past the last forced turn $[15 \mathrm{~mm}=1.60 \mathrm{~mm} ; 25 \mathrm{~mm}=1.71 \mathrm{~mm} ; 35 \mathrm{~mm}=$ $1.90 \mathrm{~mm} ; 45 \mathrm{~mm}=1.74 \mathrm{~mm} ; F(3,261)=8.27$, $\mathrm{p}<.001]$. These mean distances demonstrate the generally observed path of movement whereby, on contacting the far wall, woodlice would gradually move away and

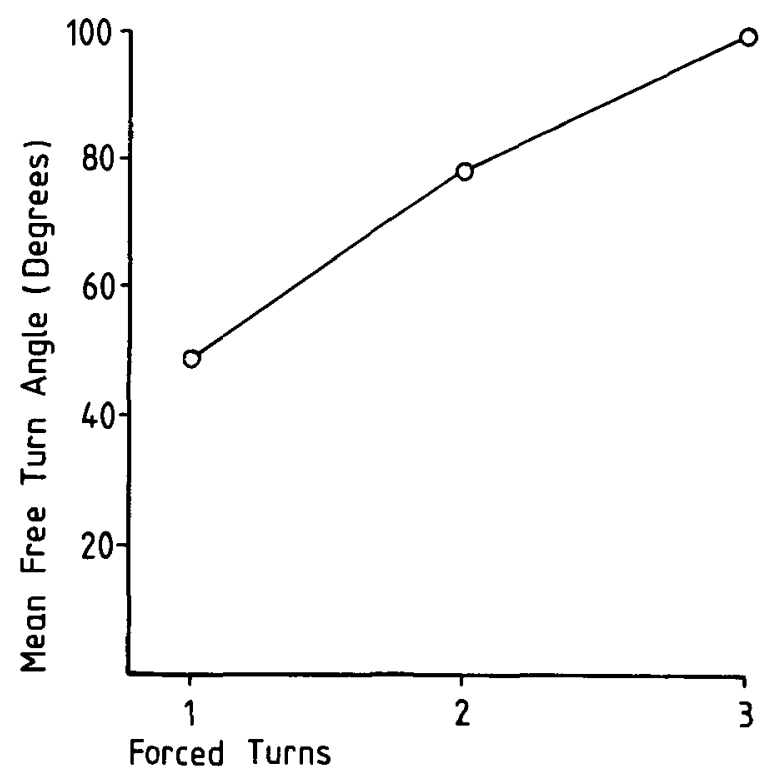

Figure 3. Effects of number of successive forced turns on freeturn angle. 
then back again on approaching the exit. The discrepancy between this pattern and the nonsignificant trend observed in Experiment 3 may have been due to differences in exitalley length and in the first point where measurements were taken.

As in Experiment 3, there was a significant (but smaller) negative correlation for all subjects between distance from the exit-alley far wall and free-turn angle $[\mathrm{r}(88)$ $=-.38, p<.01]$. Woodlice that alternated remained significantly closer to the wall $(\mathrm{n}=80$, mean $=1.66 \mathrm{~mm})$ than those that did not $[n=10$, mean $=2.38 \mathrm{~mm}, t(78)$ $=4.40, \mathrm{p}<.001]$.

The effects of greater numbers of forced turns on freeturn angle agreed with earlier reports of woodlouse alternation at T choice-points (Hughes, 1967; Sachs et al., 1965). Although, in this case, the tendency to travel close to the exit-alley far wall was not significantly affected by different numbers of forced turns, it was nevertheless related to whether or not a subject alternated and to the angle turned. Overall, the results are compatible with there being a BALM-related bias to turn in the direction opposite to that of a forced turn, which becomes evident before a free-turn choice is actually made. In addition, they establish a relationship between number of forced turns and free-turn angle which does not appear to have been previously demonstrated for any arthropod species.

\section{EXPERIMENT 5}

The results of the preceding experiments support the notion of BALM's leading to closer wall contact (accompanied by increased alternation) because of a bias to turn in the direction opposite to that of the forced turn(s). This interpretation assumes that wall contact is a reflection of BALM effects rather than the determinant of free-turn angle size. However, it is conceivable that the experimental conditions directly encouraged closer enduring thigmotaxis-based wall contact and consequently the angle turned at the runway exit. In other words, the closer the subjects were to the exit-alley far wall, the greater may have been their tendency to continue following it at the exit. The strength of this tendency would therefore have appeared as the size of the angle turned. If this were so, switching contact from one wall to another should obliterate any influence of the first wall, resulting in turning in the direction of the second wall.

Experiment 5 was designed to force subjects three times in one direction and then once in the opposite direction prior to a free turn. As woodlice are carried forward to some extent by their own momentum and do not completely turn immediately after losing contact with a wall, this procedure should bring each subject near the far wall of the exit alley. If alternation were due to wall following alone, the subjects should follow this wall and then turn in its direction at the exit, that is, alternate with respect to the last forced turn. However, if BALM were responsible, the influence of the last forced turn should be insufficient to counteract the cumulative effects of the preceding turns. On reaching the exit, subjects should therefore alternate with respect to these earlier turns, that is, they would turn in the same direction as the last forced turn. They should also remain further away from the far wall of the exit alley than woodlice that had experienced fewer forced turns in the direction opposite to that of the last turn. Overall, when negotiating the last forced turn, the weaker any BALM-related bias to turn in the direction opposite to that of the preceding forced turns, the closer should be the contact with the exit-alley far wall and the greater the angle of alternation with respect to the last forced turn.

\section{Method}

Subjects and Apparatus. The subjects were 60 naive woodlice. The Perspex runways were of the same width and depth as those used in the earlier experiments, either with three consecutive $90^{\circ}$ turns in one direction followed by a fourth $90^{\circ}$ turn in the opposite direction or with one turn in one direction and a second in the opposite direction. For all runways, the alley preceding the first forced turn was $20 \mathrm{~mm}$ long. Lengths of the alley preceding the last forced turn and the exit were $\mathbf{4 0}$ and $30 \mathrm{~mm}$, respectively. All other alley lengths were $30 \mathrm{~mm}$. The video-recording equipment was the same as that used in the preceding experiments.

Procedure. Thirty woodlice were individually introduced into the runway with four forced turns (half forced LLLR, half forced RRRL). A further 30 animals were introduced into the runway with two turns (half forced LR, half forced RL). Each subject's freeturn angle was noted and, by means of the video recording and playback procedure described for the preceding experiments, distances from the exit-alley far wall were measured $0,10,20$, and $30 \mathrm{~mm}$ past the last forced turn.

\section{Results and Discussion}

Free-turn angle was significantly smaller following three forced turns in the direction opposite to that of a fourth (mean $=-19.6^{\circ}$ ) than following one forced turn in each direction [mean $=29.57^{\circ} ; \mathrm{t}(58)=2.58$, $\mathrm{p}<.02]$. The negative angle recorded for the former group of 30 animals indicates their significant tendency to turn in the same direction as the last forced turn (21, $\mathrm{p}<.02$ ). This is contrary to the performance of the latter subjects, which alternated significantly $(22, \mathrm{p}<.01)$.

Distances from the exit-alley far wall were significantly related to both the forced-turn procedure $[\mathrm{F}(1,58)=$ $21.81, \mathrm{p}<.001]$ and the points where measurements were taken $[F(3,174)=23.30, p<.001]$. However, these effects are more appropriately considered in terms of a significant interaction between them, outlined in Figure $4[F(3,174)=12.14, p<.001]$. Woodlice forced LLLR and RRRL were furthest from the far wall immediately following the last forced turn but then moved closer while walking along the exit alley $[F(3,87)=32.98$, $p<.001]$. However, distances from the far wall did not change significantly for woodlice forced LR or RL $[F(3,87)=1.53, p<.1]$, which, overall, as already shown, traveled closer to the wall. Woodlice that repeated with respect to the last forced turn kept significantly further away from the wall $(n=29$, mean $=3.00 \mathrm{~mm})$ than 


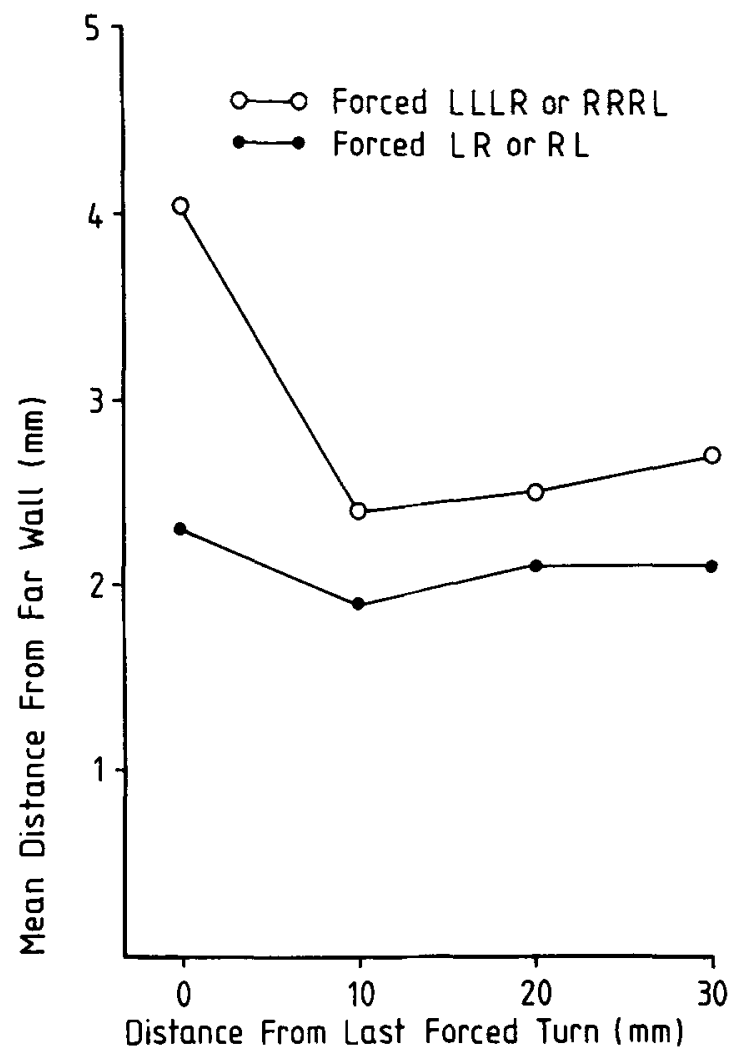

Figure 4. Distances from the exit-alley far wall measured at four points past the last forced turn for woodlice forced three successive times in one direction and a fourth in the opposite direction (LLLR or RRRL) and for those forced once in both directions (LR or RL).

those that alternated $[\mathrm{n}=31$, mean $=5.86 \mathrm{~mm} ; \mathrm{t}(58)=$ $5.86, \mathrm{p}<.001]$. There was also a significant negative correlation for all subjects between distance from the far wall and free-turn angle $[\mathrm{r}(58)=-.64, \mathrm{p}<.001]$.

This experiment showed that the effects of three forced turns in one direction were still sufficiently powerful to overcome the effects of a fourth turn in the opposite direction. Consequently, by repeating the last forced turn at the choice area, subjects alternated with respect to the three preceding forced turns. As expected from a BALM interpretation, they also remained further away from the exit-alley far wall than woodlice forced once in each direction. The latter subjects also showed no significant tendency to vary their distance from the wall, suggesting that the second forced turn cancelled the BALM effects of the first. Overall, the results of this experiment provide strong evidence for BALM rather than thigmotaxis as the principle mechanism underlying woodlouse alternation. They support the view that the apparent wall following observed in earlier experiments is not a determinant of alternation but is merely a consequence of BALM effects.

\section{EXPERIMENT 6}

If alternation arises from BALM rather than from wall contact, the antennae should have little involvement in the phenomenon. This is confirmed by the observation that removal of the antennae in $P$. scaber had no effect on sequential alternation at successive T choice-points (Hughes, 1978). There is also no evidence that receptors sensitive to contact are present on the antennae (Friedlander, 1964), even though woodlice placed in a receptacle usually move so that one antenna remains in contact with the wall (Waloff, 1941). Nevertheless, in the preceding experiments, each subject appeared to vigorously "explore" with the antennae the wall it was closest to while traveling along the exit alley. It is therefore possible that, through kinesthetic rather than tactile feedback, the antennae were involved in maintaining appropriate distances from the far wall. As these distances reflect prechoice BALM effects but do not directly influence alternation, the antennae should not determine free-turn angle size. This final experiment examined the importance of the antennae in controlling distance from the exit-alley far wall as well as the angle subsequently turned.

\section{Method}

Subjects and Apparatus. The subjects were 96 naive woodlice. The apparatus comprised the runway with a $90^{\circ}$ forced turn, as described in Experiment 1, and the video-recording equipment described in the preceding experiments.

Procedure. The subjects were immobilized in groups of six with gaseous carbon dioxide. By means of fine dissecting forceps, the left second antenna was removed (L) from 24 animals, the right was removed (R) from another 24, and both were removed (L\&R) from a further 24; the remainder were immobilized and handled, but neither antenna was removed (0). The following day, each subject was introduced into the runway and its free-turn angle noted. By means of the video-recording and playback procedure described earlier, distances from the exit-alley far wall were measured at points $0,10,20$, and $30 \mathrm{~mm}$ past the forced turn. On the stopped image at $30 \mathrm{~mm}$, it was also noted in each case whether the subject was parallel with the alley walls or slightly oriented toward or away from the far wall.

\section{Results and Discussion}

Because of the removal of a left or a right antenna in two groups, the two forced-turn directions were also included in the ANOVAs. Free-turn angle was unaffected by either direction of forcing [ forced $\mathrm{L}$ mean $=52.96^{\circ}$, forced $R$ mean $\left.=61.40^{\circ} ; F(1,88)=0.86, p>.25\right]$ or removal of antennae $\left[0\right.$ mean $=64.35^{\circ}, \mathrm{L}$ mean $=$ $52.92^{\circ}, \mathrm{R}$ mean $=68.98^{\circ}, \mathrm{L} \& \mathrm{R}$ mean $=42.46^{\circ} ; \mathrm{F}(3,88)$ $=1.71, \mathrm{p}>.1 \mathrm{]}$. Although significant numbers of subjects (out of 24) with neither, one, or both antennae removed alternated $(0=24, \mathrm{p}<.001 ; \mathrm{L}=18, \mathrm{p}<.03$; $\mathrm{R}=23, \mathrm{p}<.002 ; \mathrm{L} \& \mathrm{R}=22, \mathrm{p}<.002]$, there were no significant differences between them $\left[\chi^{2}(2)=2.00\right.$, $\mathrm{p}>.1$; results of the $L$ and $R$ groups were combined to avoid invalidation of the chi-square test by excessively low expected frequencies; Siegel, 1956].

As there were no obvious systematic between-groups differences in distances from the exit-alley far wall measured at four points past the forced turn, average distances were calculated for individual subjects. These averages were significantly affected by both direction of forcing $[F(1,88)=7.83, p<.01]$ and removal of antennae 


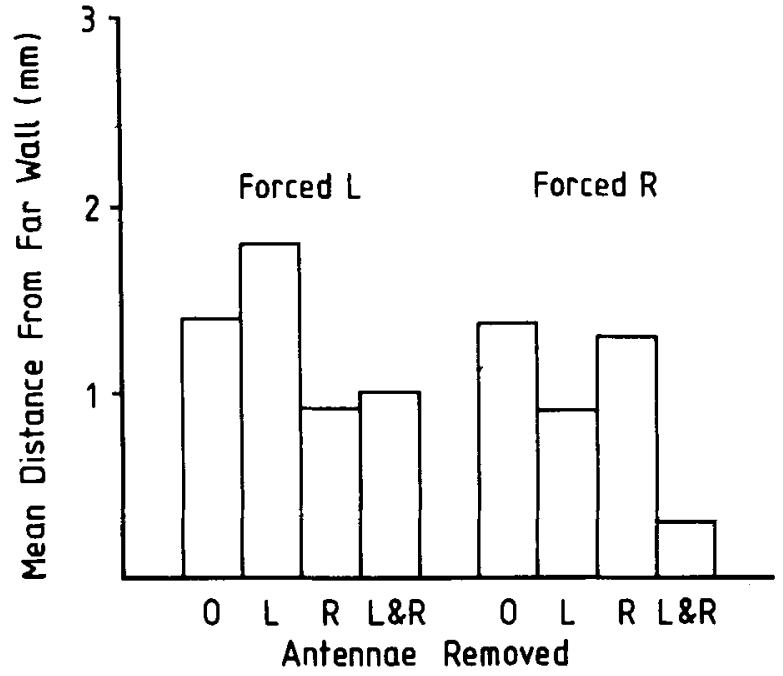

Figure 5. Distances from the exit-alley far wall in woodlice forced left or right after removal of one or both antennae.

$[F(3,88)=12.09, p<.001]$. However, these effects must be considered in terms of a significant interaction between them $[F(3,88)=9.12, p<.001$; see Figure 5]. Figure 5 shows that, when forced left, subjects with their left antenna removed remained further away from the wall than any other group. Conversely, subjects with the right antenna removed remained closest to this wall. When forced right, the reverse situation prevailed for these two groups, indicating that unilateral removal of antennae produced movements toward the wall on the same side as the loss. Subjects with both antennae removed remained closer than most other groups to the far wall irrespective of forced-turn direction. They were also closer after a right forced turn, possibly because of some inadequately controlled extraneous influence, such as bilateral illumination.

Although there was no significant correlation for all subjects between average distance from the exit-alley far wall and free-turn angle $[\mathrm{r}(94)=-.14, \mathrm{p}>.1]$, woodlice that alternated remained significantly closer to the wall $(n=87$, mean $=1.08 \mathrm{~mm})$ than those that did not $[\mathrm{n}=9$, mean $=1.67 \mathrm{~mm} ; \mathrm{t}(94)=2.34, \mathrm{p}<.05]$.

The numbers of woodlice in each group that were oriented toward, away from, or parallel with the exit-alley far wall $30 \mathrm{~mm}$ past the forced turn can be seen in Table 1. Significant numbers of subjects with one (31, $\mathrm{p}<.05$ ) or both antennae removed were oriented toward

Table 1

Numbers of Woodlice With Neither, One, or Both Antennae Removed that were Oriented Toward, Away From, or Parallel With the Exit-Alley Far Wall, $30 \mathrm{~mm}$ Past the Forced Turn

\begin{tabular}{cccc}
\hline Group* & Oriented Toward & Oriented Away & Parallel With \\
\hline 0 & 8 & 2 & 14 \\
L & 18 & 5 & 1 \\
R & 13 & 11 & 0 \\
L\&R & 18 & 6 & 0 \\
\hline
\end{tabular}

$*_{n}=24$. the far wall $(18, p<.03)$, and hence the direction of an alternating turn. This was not so for intact animals (8, $p>.1)$. Differences between these numbers was also significant $\left[\chi^{2}(2)=9.19, \mathrm{p}<.02\right]$. However, there was no significant difference in free-turn angle between woodlice that were oriented toward $\left(n=57\right.$, mean $\left.=60.20^{\circ}\right)$ and those that were oriented away from or parallel with the wall $\left[\mathrm{n}=39\right.$, mean $\left.=52.77^{\circ} ; \mathrm{t}(94)=.77, \mathrm{p}>.2\right]$. This result confirms the lack of a relationship between orientation and free-turn angle reported in Experiment 2.

Although uni- and bilateral removal of antennae produced changes in both the distance traveled from and orientation to the exit-alley far wall, these changes were unrelated to turns made at the choice area. Nevertheless, they appear to determine how BALM effects modify behavior occurring between forced and free turns.

\section{GENERAL DISCUSSION}

Experiments 1, 4, and 6 established that although freeturn angle in $P$. scaber was increased with greater forcedturn angles and the number of forced turns in one direction, loss of one or both antennae had no effect on the response. The remaining experiments revealed a relationship between distance from (but not orientation to) the exit-alley far wall and free-turn angle size. This finding is consistent with the notion that BALM effects cause subjects to travel closer to the wall because of greater locomotor force of the inner legs which were more "rested" during negotiation of the forced turn(s). Although the antennae were not necessary for BALM to influence woodlouse alternation, thereby confirming an earlier observation (Hughes, 1978), they were clearly involved in how BALM effects were manifested in behavior occurring between forced and free turns.

Because of the pattern of exit-alley movement and the observation that free-turn angle depended not on the last forced turn but on the cumulative effects of preceding turns in the opposite direction, Experiment 5 ruled out the possibility that woodlouse alternation was due to thigmotaxis-related wall-following behavior. Turning in the direction of additional unilateral forced walking reported by Beale and Webster (1971) was therefore almost certainly due to the effects of BALM.

It is concluded that turn alternation in woodlice mostprobably arises from the effects of turn-elicited BALM. The BALM-induced tendency to "correct" following forcing, which was evident even before a free turn (as shown by distances from the exit-alley far wall), suggests a more appealing mechanistic basis for woodlouse alternation than, for example, the short-term memory explanation proposed for pill bugs (Kupferman, 1966). More detailed comparative research is obviously required to determine whether or not turn alternation in crustaceans, such as woodlice, is a different phenomenon from that in insects (as suggested by Kupferman, 1966). The level of control of BALM must also be established as it is not yet clear whether it has a neural basis or whether it merely 
arises from some other influence, such as muscle fatigue. If neural factors are responsible, fine-grain analyses of leg movements during turning and immediately afterward could help determine relative contributions to BALM of amplitude and phase-modulation locomotor control systems (Stein, 1977) as well as the importance of peripheral versus central neural control.

\section{REFERENCES}

Beale, I. L., \& Webster, D. M. (1971). The relevance of leg-movement cues to turn alternation in woodlice (Porcellio scaber). Animal Behaviour, 19, 353-356.

Corballis, M. C., \& Beale, I. L. (1983). The ambivalent mind. Chicago: Nelson-Hall.

Dember, W. N. (1961). Alternation behavior. In D. W. Fiske \& S. R. Maddi (Eds.), Functions of varied experience. Homewood, IL: Dorsey Press.

DinGle, H. (1961). Correcting behavior in boxelder bugs. Ecology, 42, 207-211.

Dingle, H. (1964a). Correcting behaviour in mealworms (Tenebrio) and the rejection of a previous hypothesis. Animal Behaviour, 12, 137-139.

DingLe, H. (1964b). Further observations on correcting behaviour in boxelder bugs. Animal Behaviour, 12, 116-124.

DingLe, H. (1965). Turn alternation by bugs on causeways as a delayed compensatory response and the effects of varying visual inputs and length of straight path. Animal Behaviour, 13, 171-177.

Friedlander, C. P. (1964). Thigmokinesis in woodlice. Animal Behaviour, 12, 164-174.

Grosslight, J. H., \& HaRrison, P. C. (1961). Variability of response in a determined turning sequence in the meal worm (Tenebrio molitor): An experimental test of alternative hypotheses. Animal Behaviour, 9, 100-103.

Grosslight, J. H., \& TICKNOR, D. (1953). Variability and reactive in- hibition in the mealworm as a function of determined turning sequence. Journal of Comparative \& Physiological Psychology, 46, 35-38.

HANSELL, M. H., \& AITKEN, J. J. (1977). Experimental animal behavior: A selection of laboratory exercises. Glasgow: Blackie.

Hughes, R. N. (1967). Turn alternation in woodlice (Porcellio scaber). Animal Behaviour, 15, 282-286.

Hughes, R. N. (1978). Effects of blinding antennectomy, food deprivation and simulated natural conditions on alternation in woodlice (Porcellio scaber). Journal of Biological Psychology, 20, 35-40.

Hurley, D. E. (1950). New Zealand terrestrial isopods. Tuatara, 3, $115-127$.

JENSEN, D. D. (1959). A theory of the behavior of Paramecium aurelia and behavioral effects of feeding, fission, and ultraviolet microbeam iradiation. Behaviour, 15, 82-122.

Kupferman, I. (1966). Turn alternation in the pill bug (Armadillidium vulgare). Animal Behaviour, 14, 68-72.

Pardi, L., \& PAPI, F. (1961). Kinetic and tactic responses. In T. H. Waterman (Ed.), The physiology of crustacea (Vol. 2). New York: Academic Press.

SACHS, L. B., Klopfer, F. D., \& MorRow, J. E. (1965). "Reactive inhibition" in the sowbug. Psychological Reports, 17, 739-743.

Shinkman, P. G., \& HerTzler, D. R. (1964). Maze alternation in the planarian, Dugesia tigrina. Psychonomic Science, 1, 407-408.

SIEGEL, S. (1956). Nonparametric statistics for the behavioral sciences. New York: McGraw-Hill.

STEIN, P. S. G. (1977). A comparative approach to the neural control of locomotion. In G. Hoyle (Ed.), Identified neurons and behavior of arthropods. New York: Plenum Press.

WALOFF, N. (1941). The mechanisms of humidity reactions of terrestrial isopods. Journal of Experimental Biology, 18, 115-135.

WILSON, M. M., \& FowLER, H. (1976). Variables affecting alternation behavior in the cockroach, Blatta orientalis. Animal Learning \& Behavior, 4, 490-494.

(Manuscript received July 20, 1984; revision accepted for publication August 22, 1985.) 